\title{
Improvement and Taste Symmetry Breaking for Staggered Quarks * $\dagger$
}

\author{
E. Follana ${ }^{\text {a }}$, C. Davies ${ }^{\text {a }}$, A. Hart ${ }^{\text {b }}$, P. Lepage ${ }^{\text {c }}$, Q. Mason ${ }^{c}$, H. Trottier ${ }^{\text {d }}$, HPQCD and UKQCD \\ collaborations. \\ a Department of Physics and Astronomy, University of Glasgow, Glasgow G12 8QQ, UK. \\ ${ }^{\mathrm{b}}$ School of Physics, University of Edinburgh, Edinburgh EH9 3JZ, UK. \\ cNewman Laboratory for Elementary-Particle Physics, Cornell University, Ithaca NY 14853, USA. \\ ${ }^{\mathrm{d}}$ Department of Physics, Simon Fraser University. Burnaby, British Columbia V5A 1S6, CANADA.
}

We compare several improved actions for staggered quarks. We study the effect of improvement on the taste changing interactions by calculating the splitting in the pion spectrum. We investigate the effect of the improvement on some topological properties.

\section{Introduction}

It is well known that the naive discretization of quark fields in lattice QCD results in the generation, in dimension $d$, of $2^{d}$ species of fermions, which we will call 'tastes'. The theory also contains taste-changing interactions. This implies, for example, that the spectrum of the theory will have several non equivalent versions of the usual hadrons.

The staggered fermion formulation, in 4 dimensions, reduces the number of doublers to 4 . The taste-changing interactions are perturbative, and can be reduced by improving the action [1234]. The breaking of taste symmetry is most clearly seen in the splitting of the pion spectrum. In the staggered formulation there are 16 different pions, which are grouped in 5 (approximate) multiplets. Calculating the splitting between the pion masses

\footnotetext{
*Talk presented by E. Follana. Work supported by PPARC.

${ }^{\dagger}$ The simulations for this work were carried out on a computer network running Condor at Dallas Southern Methodist University. The Condor Software Program (Condor) was developed by the Condor Team at the Computer Sciences Department of the University of WisconsinMadison. All rights, title, and interest in Condor are owned by the Condor Team.

We thank K. Hornbostel for his useful help in connection with this work.

We thank Anna Hasenfratz for her assistance in implementing the HYP action.
}

in the different multiplets gives a measure of the breaking of taste symmetry.

\section{Improved Staggered Actions and Taste Symmetry Breaking}

We have investigated the effect of taste symmetry breaking interactions for several improved staggered action, some of them inspired by perturbative calculations, others by perfect action ideas.

First we have the asqtad action, which removes taste-changing interactions at tree level 123 and is fully $a^{2}$ improved. If we don't include the Lepage and Naik terms, we obtain the so-called fat7 action, which can be viewed as the unimproved staggered action calculated on fattened (and tadpole improved) links. We can iterate this procedure, using the fattened links obtained in a step of fat7 to build asqtad or fat7 again (and for these we use the obvious notation fat7xfat7, etc.) We also have the choice of projecting the fattened links back to $S U(3)$ or not.

Another action which we study is the so called hypercubic blocked (HYP) action, which can be viewed as the Kogut-Susskind action built on a different kind of fattened links [5].

We have used a set of $\approx 200$ quenched Wilson pure gauge UKQCD configurations, with a lattice size of $16^{3} \times 32$, at $\beta=5.9$ and $a \approx 0.1 \mathrm{fm}$. With 


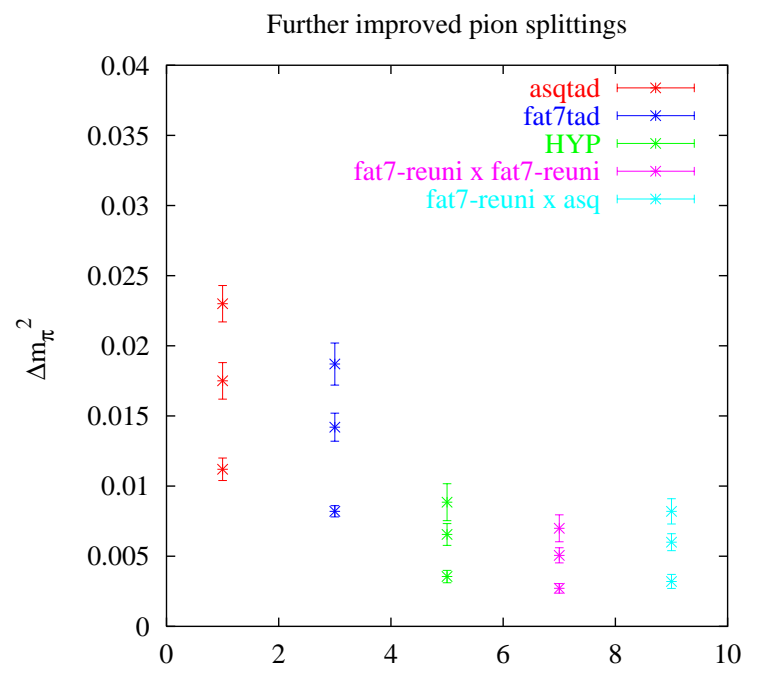

Figure 1. Pion splitting: mass squared difference between central multiplets and Goldstone pion, for several improved staggered actions

these configurations we do a quenched calculation to obtain the complete set of pions for each of the actions previously described. The fitting of pion correlators is done using a Bayesian fitting method.

In figure 1 we plot the quadratic mass deviation from the Goldstone mass in lattice units, $\Delta m_{\pi}^{2} a^{2}$, for the three central multiplets. We see that both the HYP action and the iterated fattened actions result in a considerable reduction of the splitting with respect to the asqtad or fat7 action. Furthermore, it has been noticed before [3] that the reunitarization of the fattened links has a considerable effect on the splitting. Our results agree with this [6].

\section{Improved Staggered Actions and Topol- ogy}

The usual gluonic definition of topological charge is given by

$Q=\frac{1}{32 \pi^{2}} \sum_{x} \frac{1}{32} \epsilon_{\mu \nu \sigma \tau}^{ \pm} \operatorname{Tr} U_{\mu \nu}(x) U_{\sigma \tau}(x)$ whereas the fermionic definition is

$Q=\frac{m}{n_{f}} \operatorname{tr}\left(\gamma_{5} S_{F}\right)=\frac{m^{2}}{n_{f}} \sum_{n} \frac{<n\left|\gamma_{5}\right| n>}{\lambda^{2}+m^{2}}$

where $\mid n>$ are the eigenfunctions of the Dirac operator in the given gauge field background 7 .

In the continuum the index theorem holds, and we have $Q=n^{+}-n^{-}$, where $n^{+}$is the number of positive chirality zero modes, and $n^{-}$the number of negative chirality ones. In the lattice, for a nonchiral discretization of the Dirac operator, we lose the index theorem. However, we can still hope to find a correlation between "approximate zero modes", which have a high value of the chirality $<n\left|\gamma_{5}\right| n>$, and gluonic topological charge.

In our set of quenched configurations, we first calculate the topological charge using the gluonic definition after a cooling procedure. We also calculate the 20 lowest lying eigenvectors for both the unimproved staggered and for several of the improved actions. In figure 2 we show the chirality for the first 20 eigenvalues of the Dirac operators in a configuration where, should the index theorem hold, the first 12 eigenvectors would have been chiral. We can see a clear qualitative difference as we improve the action, with the appearance of approximate zero modes in the required number needed to produce the given topological charge.

To get a more quantitative measure, we calculate $\sum_{n}<n\left|\gamma_{5}\right| n>$ over the 20 eigenvectors. If the lowest eigenmodes are approximately chiral (and small), and the rest are not, this quantity should be approximately equal to the topological charge for a fairly large range in $m$. In figure 3 we show the correlation between this quantity and the gluonic topological charge for the unimproved staggered and for the fat7xasqtad actions. We can see that the two definitions agree quite well for the improved action. In the unimproved case there is still a correlation, but the two definitions give very different results for the topological charge.

\section{REFERENCES}

1. G. Peter Lepage, Phys.Rev. D59 (1999) 074502 . 


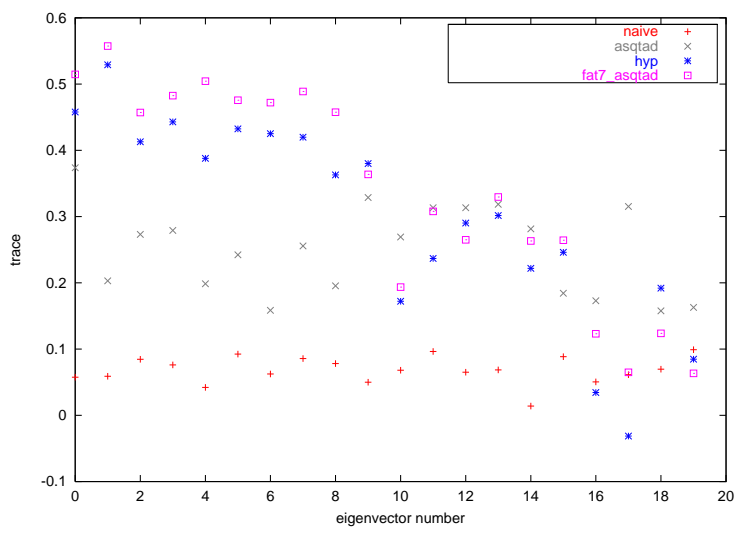

Figure 2. Chirality of the lowest eigenvectors of the Dirac operator.

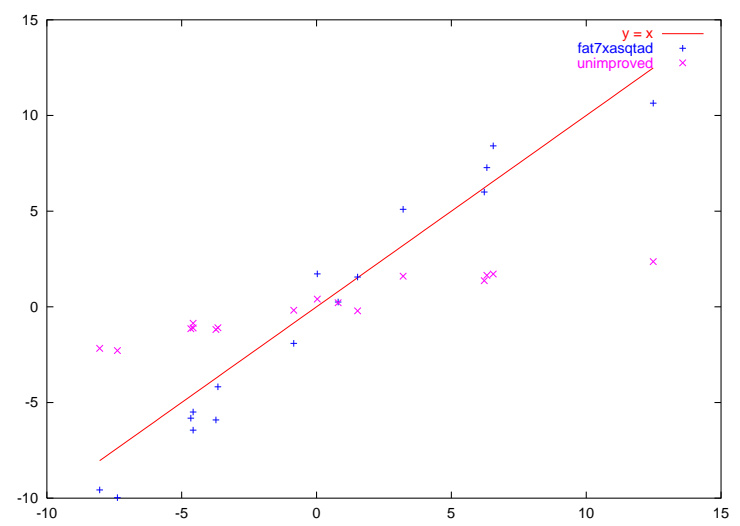

Figure 3. Correlation between the gluonic and the fermionic definitions of topological charge. The $\mathrm{x}$ symbols correspond to the unimproved staggered action, whereas the + ones correspond to the fat7xasqtad action. The straight line represents $\mathrm{y}=\mathrm{x}$.
2. The MILC collaboration: C. Bernard, T. Burch, T.A. DeGrand, C.E. DeTar, S. Gottlieb, U.M. Heller, J. Hetrick, K. Orginos, R.L. Sugar, D. Toussaint, Phys.Rev. D61 (2000) 111502.

Tom Blum, Carleton DeTar, Steven Gottlieb, Urs M. Heller, James E. Hetrick, Kari Rummukainen, R.L. Sugar, Doug Toussaint, Matthew Wingate Phys.Rev. D55 (1997) 1133-1137.

3. Kostas Orginos, R.L. Sugar, Doug Toussaint, Phys.Rev. D60 (1999) 054503.

4. Q. Mason et al, this proceedings.

5. F. Knechtli, A. Hasenfratz, Phys. Rev. D63 (2001) 114502.

6. C. Davies, E. Follana, K. Hornbostel, P. Lepage, Q. Mason, H. Trottier, in preparation.

7. J. Smit and J.C. Vink, Nucl. Phys. B 303 (1988), 36;

J. C. Vink, Nucl. Phys. B 307 (1988). 